\title{
Effects of siRNA-mediated suppression of HPV-11 L1 expression on the proliferation and apoptosis of vaginal epithelial cells
}

\author{
JUAN ZENG $^{1^{*}}$, SHUMEI YANG ${ }^{*}$, XIAORUI WANG ${ }^{2}$, YAN GAO $^{3}$ and MEI ZHANG ${ }^{1}$ \\ ${ }^{1}$ Department of Obstetrics and Gynecology, The Second Affiliated Hospital of Xi'an Medical University, Xi'an, \\ Shaanxi 710038; ${ }^{2}$ Department of Oral Medicine, The 323rd Hospital of The People's Liberation Army, Xi'an, Shaanxi 710032; \\ ${ }^{3}$ Nursing Department, The Second Affiliated Hospital of Xi'an Medical University, Xi'an, Shaanxi 710038, P.R. China
}

Received September 11, 2015; Accepted October 28, 2016

DOI: $10.3892 /$ etm.2017.4120

\begin{abstract}
The aim of the present study was to investigate the effects of human papillomavirus (HPV) infection on the gynecological disease of vaginitis and to demonstrate how the small interfering RNA (siRNA) method may be used for HPV prevention in the clinic. Human vaginal epithelial cells were transfected with HPV-11 L1 expression vector and siRNA-HPV-11 L1 vectors and a control group was transfected with scrambled siRNA. Cell proliferation in each group was analyzed using the MTT assay and the expression of apoptosis-associated proteins was measured by western blot analysis. Compared with the control group, HPV-11 L1 mRNA and protein levels were significantly increased following transfection with the HPV-11 L1 expression vector in cells $(\mathrm{P}<0.05)$, but this result was significantly reversed by silencing of HPV-11 L1 ( $<<0.05)$. In addition, cell proliferation in the HPV-11 group was lower than that in the control group; however, cell proliferation was significantly increased in cells transfected with silenced L1 compared with that in the control group $(\mathrm{P}<0.05)$. Furthermore, silencing of HPV-11 L1 significantly decreased caspase-3 and caspase-9 expressions in cells, whereas the expression was increased in the HPV-11 L1 group $(\mathrm{P}<0.05)$. The present study suggested that siRNA-mediated silencing of HPV-11 L1 may have potential therapeutic applications for treating gynecological diseases associated with HPV-11 infection.
\end{abstract}

\section{Introduction}

Vaginitis is a common gynecological problem, which is induced by inflammation or infection of the vagina (1). The

Correspondence to: Professor Mei Zhang, Department of Obstetrics and Gynecology, The Second Affiliated Hospital of Xi'an Medical University, 167 Fangdong Road, Xi'an, Shaanxi 710038, P.R. China

E-mail: zhangmei985_21@126.com

\section{*Contributed equally}

Key words: vaginitis, human papillomavirus, vaginal epithelial cells, small interfering RNA most common symptoms of vaginitis are itching, burning and discharge. Statistics show that up to $75 \%$ of married women present with vaginitis, cervicitis or endometritis at a certain stage (2). At present, vaginitis is typically treated by pharmaceutical, antibiotic and endocrine therapy $(3,4)$. However, due to the complex pathogenic mechanisms of vaginitis and the high risk of relapse, it is required to further explore methods for diagnosis and treatment to improve therapeutic effects (5).

The human papillomavirus (HPV) is a multifunctional DNA-binding protein that is associated with various sexually transmitted diseases and divided into high-risk and low-risk groups according to its oncogenic risk $(6,7)$. High-risk HPVs, including HPV-16 and HPV-18, are able to induce cervical cancers and also contribute to a high proportion of other anogenital and oropharyngeal cancers (8), whereas low-risk HPVs, such as HPV-6 and HPV-11, induce anogenital warts and recurrent respiratory papillomatosis (9). Previous studies have focused on the association between HPV infection and gynecologic disease, and have demonstrated that numerous women with vaginitis also have HPV infection $(10,11)$. For example, the study by Weihua (12) used fluorescent quantitative polymerase chain reaction (qPCR) to demonstrate that HPV-11 levels were high in patients with vaginitis, and a meta-analysis by Banura et al (13) showed that women with HPV infections were at a greater risk of developing bacterial vaginitis. However, few studies have explored the association between HPV-11 L1 protein and vaginitis by using small interfering RNA (siRNA). A previous study reported that the targeting of E6-associated protein which mediated by siRNA in cervical specimens was a potential therapeutic strategy for women infected with HPV (14).

In the present study, the expression of HPV-11 L1 in human epidermal carcinoma cells derived from vaginal epithelial cell lines was evaluated using siRNA-mediated gene silencing. Comprehensive experimental methods were used to analyze the effects of HPV-11 L1 protein expression and vaginal epithelial cell proliferation. The present study aimed to investigate the possible applications of siRNA silencing in analyzing the correlation between HPV-11 L1 expression and gynecological diseases.

\section{Materials and methods}

Cell culture and HPV-11 L1 transfection. Vaginal epithelial cells (A341; American Type Culture Collection, Manassas, 
VA, USA) were cultured in Dulbecco's modified Eagle's medium (DMEM; GE Healthcare Life Sciences; Hyclone, Logan, UT, USA) supplemented with $10 \%$ fetal bovine serum (FBS; Gibco; Thermo Fisher Scientific, Inc., Waltham, MA, USA) and antibiotics (0.05 U/ml penicillin and $0.05 \mathrm{U} / \mathrm{ml}$ streptomycin; Sigma-Aldrich; Merck Millipore, Darmstadt, Germany), and $10 \mathrm{mM}$ HEPES (Sigma-Aldrich) at $37^{\circ} \mathrm{C}$.

HPV-11 L1 expressing vector (Sigma-Aldrich; Merck Millipore) was transfected into vaginal epithelial cells using the FuGENE 6 transfection reagent (Invitrogen; Thermo Fisher Scientific, Inc.). In brief, the HPV-11 L1 plasmid was mixed with FuGENE 6 reagent and OPTI-MEM medium (Invitrogen; Thermo Fisher Scientific, Inc.) to form a transfection agent-plasmid mixture. Subsequently, the mixture was added to each well of a 96-well plate and a 6-well plate containing OPTI-MEM and cells. The transfection was allowed to proceed for $12 \mathrm{~h}$.

HPV-11 L1 siRNA transfection. Following $24 \mathrm{~h}$ of serum starvation, cells were transfected using Lipofectamine ${ }^{\circledR} 3000$ (Thermo Fisher Scientific, Inc.) reagent according to the manufacturer's protocol. In brief, 0.05-1.5 $\mu \mathrm{g} / \mathrm{ml} \mathrm{HPV-11}$ L1 siRNAs or scramble siRNA as control that synthesized by Guangzhou RiboBio Co., Ltd. (Guangzhou, China) and $12 \mu \mathrm{g} / \mathrm{ml}$ Lipofectamine ${ }^{\circledR} 3000$ were diluted in serum-free DMEM. Following $30 \mathrm{~min}$ of incubation at $37^{\circ} \mathrm{C}$, the complexes were added dropwise to each culture dish and incubated at $37^{\circ} \mathrm{C}$ in an atmosphere containing $5 \% \mathrm{CO}_{2}$ for $12 \mathrm{~h}$. Following transfection, the cells were cultured in serum-free DMEM.

Reverse transcription (RT)-qPCR. After $48 \mathrm{~h}$ of treatment, mRNA was extracted from vaginal epithelial cells using TRIzol reagent (Sigma-Aldrich; Merck Millipore) as previously described (12), and was treated with RNase-free DNase I (cat. no. EN0521; Thermo Fisher Scientific, Inc.) (15). Micro-spectrophotometer SMA 4000 (Merinton Instrument, Inc., Ann Arbor, MI, USA) was used to measure the concentration and purity of RNA. Purified RNA at a concentration of $0.5 \mu \mathrm{g} / \mathrm{ml}$ combined with nuclease-free water was used for complementary cDNA synthesis using the PrimeScript 1st Strand cDNA Synthesis kit (cat. no. 6210A; Takara Biotechnology Co., Ltd., Dalian, China). The PCR amplification reaction was performed using $10 \%$ of the first chain cDNA liquid. Expression in target vaginal epithelial cells was detected in an Eppendorf Mastercycler (Brinkmann Instruments, Inc., Westbury, NY, USA) using the SYBR ExScript RT-qPCR kit (cat. no. DRR035A; Takara Biotechnology Co., Ltd.). The total reaction system was $20 \mu \mathrm{l}$ in volume, consisting of the following: $1 \mu \mathrm{l}$ cDNA produced via RT outlined above; $10 \mu \mathrm{l}$ SYBR Premix EX Taq, $1 \mu \mathrm{l}$ each of the primers $(10 \mu \mathrm{M})$; and $7 \mu \mathrm{l}$ double distilled $\mathrm{H}_{2} \mathrm{O}$. PCR was performed as follows: Denaturation at $50^{\circ} \mathrm{C}$ for $2 \mathrm{~min} ; 95^{\circ} \mathrm{C}$ for $10 \mathrm{~min}$; followed by 45 cycles of $95^{\circ} \mathrm{C}$ for $10 \mathrm{sec}$, and $60^{\circ} \mathrm{C}$ for $1 \mathrm{~min}$. Each real-time PCR was carried out in triplicate on the ABI PRISM 7500 Real-time PCR System (Applied Biosystems; Thermo Fisher Scientific, Inc.). Following the completion of PCR, melting curve analysis of amplification products was performed to confirm that only one product was detected and amplified. Data were analyzed according to the
$2^{-\Delta \Delta \mathrm{Cq}}$ method (16), GAPDH was used as the internal control. Primers were designed as follows: HPV-11 L1, forward, 5'-GCTCAGGGACATAACAATGG-3' and reverse 5'-CGT CCAAGGGGAAACTGATC-3'; and GAPDH forward, 5'-TATGATGATATCAAGAGGGTAGT-3' and reverse, 5'-TGTATCCAAACTCATTGTCATAC-3'.

HPV-11 L1 protein expression ELISA assay. The L1 protein sample was transferred into a 96-well ELISA plate and diluted with $0.05 \mathrm{M}$ carbonate buffer solution up to $15-20 \mu \mathrm{g} / \mathrm{ml}$ and stored overnight at $4^{\circ} \mathrm{C}$. The plate was subsequently washed with PBS and 3\% bovine serum albumin (BSA; cat no. A7906, Sigma-Aldrich) was used to block the plate (17). Blocking solution was removed and HPV-11 L1 antibody (cat. no. sc66145, Santa Cruz Biotechnology, Inc., Dallas, TX, USA) was diluted to 1:5,000 and added to the wells. The plate was washed with PBS three more times prior to the addition of 1:1,000 diluted horseradish peroxidase-labeled goat anti-mouse immunoglobulin G (cat. no. sc2386; Santa Cruz Biotechnology, Inc.) and incubated at $37^{\circ} \mathrm{C}$ for $1 \mathrm{~h}$. The plate was washed again with $\mathrm{PBS}$ and stained with tetramethylbenzidine chromogenic solution and $2 \mathrm{M} \mathrm{H}_{2} \mathrm{SO}_{4}$ was subsequently added to terminate the reaction. A microplate reader (MR-960; BioBase; Qiagen GmbH, Hilden, Germany) was used to measure the optical density (OD) value at $450 \mathrm{~nm}$; three replicates of the experiment were performed and the average value of three tests was calculated. The obtained OD values were calculated using the formula $\left(\mathrm{OD}_{\text {sample }}-\mathrm{OD}_{\text {blank }}\right) /\left(\mathrm{OD}_{\text {negative }}-\mathrm{OD}_{\text {blank }}\right)$. A calculated value of $\geq 2.1$ indicated a positive reaction.

Cell viability assay. Vaginal epithelial cells were seeded in 96-well culture plates and transfected with HPV-11 L1 siRNA or siRNA control for $72 \mathrm{~h}$ and 7 days. The number of viable cells was determined using MTT reagent (Invitrogen, Thermo Fisher Scientific, Ltd.) according to the manufacturer's protocol (18). In brief, MTT reagent $(10 \mu \mathrm{l})$ was added to $100 \mu \mathrm{l}$ of medium and incubated at $37^{\circ} \mathrm{C}$ for $4 \mathrm{~h}$. The supernatant was removed and dimethyl sulfoxide (Sigma-Aldrich) was added to solubilize the formazan crystals. The absorbance of the medium at $570 \mathrm{~nm}$ was measured with an Elx-800 Absorbance reader (BioTek Instruments, Inc., Winooski, VT, USA).

Caspase activity assay. The Fluorescence Protease Assay kit (cat. no. 23266; Thermo Fisher Scientific, Inc.) was used to detect caspase-3 and caspase- 9 activity according to the manufacturer's protocol (19). In brief, vaginal epithelial cells were lysed in lysis buffer (Beyotime Biotechnology, Nantong, Jiangsu, China) and centrifuged at $18,000 \times \mathrm{g}$ for $10 \mathrm{~min}$ at $4^{\circ} \mathrm{C}$ and supernatants were collected. Equal amounts of protein samples $(1 \mathrm{mg} / \mathrm{ml} ; 20 \mu \mathrm{l})$ were reacted with the synthetic fluorescent substrates at $37^{\circ} \mathrm{C}$ for $4 \mathrm{~h}$ and fluorescence was measured at $405 \mathrm{~nm}$ in a microplate reader (Bio-Rad Laboratories, Inc., Hercules, CA, USA). Fold-increases in caspase-3 and caspase- 9 activity were determined using values obtained from the treatment samples divided by those from the control groups.

Statistical analysis. Experiments were performed three times independently and values are expressed as the mean \pm standard 


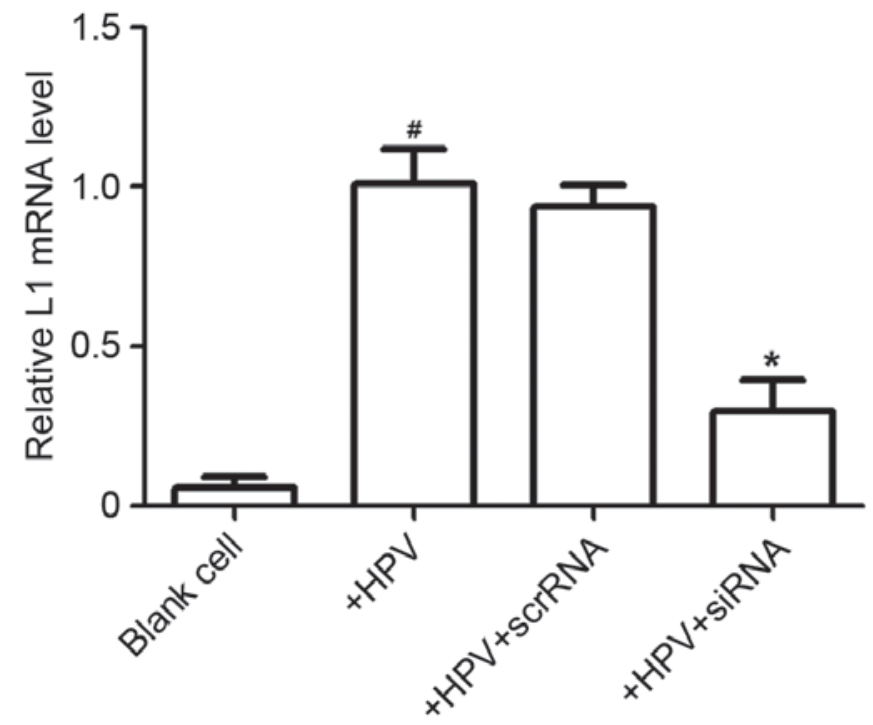

Figure 1. mRNA expression of HPV-11 L1 in vaginal epithelial cells. Following transfection of vaginal epithelial cells with HPV-11 L1, the mRNA levels of HPV-11 L1 were significantly increased compared with those in the blank control group, which was significantly reduced by siRNA. " $\mathrm{P}<0.05$ vs. the blank cells, ${ }^{\text {}} \mathrm{P}<0.05$ vs. HPV+scrRNA treated cells. HPV, human papillomavirus; siRNA, small interfering RNA; scrRNA, scrambled siRNA.

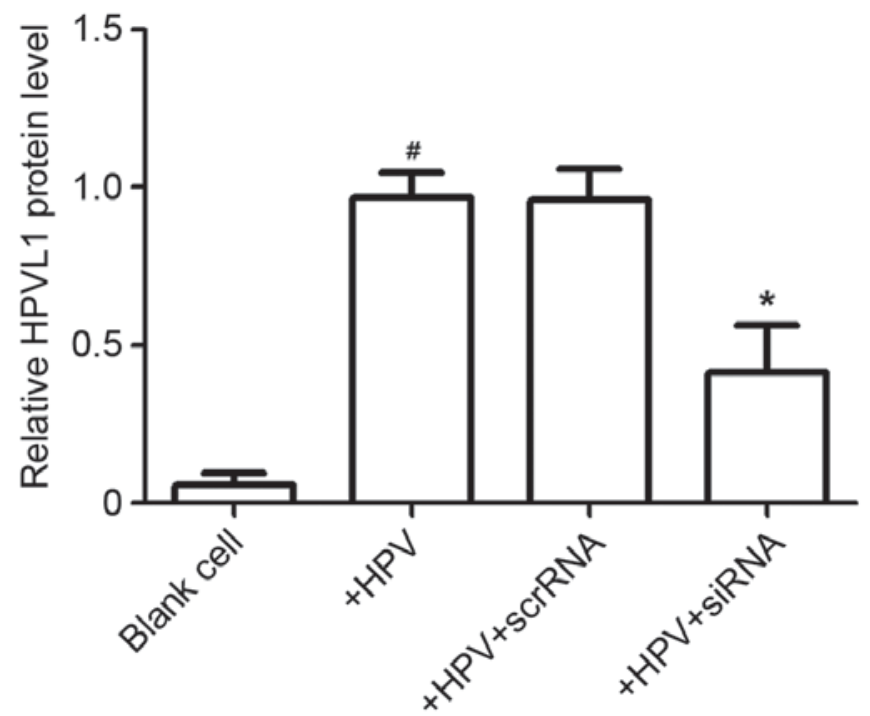

Figure 2. Expression levels of HPV-11 L1 protein in vaginal epithelial cells. Following transfection of vaginal epithelial cells with HPV-11 L1, the protein levels of HPV-11 L1 were significantly increased compared with those in the blank control group, which was significantly reduced by siRNA. ${ }^{*} \mathrm{P}<0.05$ vs. the blank cells, ${ }^{*} \mathrm{P}<0.05$ vs. the HPV+scrRNA treated cells. HPV, human papillomavirus; siRNA, small interfering RNA; scrRNA, scrambled siRNA. error of the mean. Statistical analyses were performed using GraphPad Prism 5.0 (GraphPad Software, Inc., La Jolla, CA, USA). One-way analysis of variance was performed to evaluate the difference among groups, followed by Tukey's post hoc test and $\mathrm{P}<0.05$ was considered to indicate a statistically significant difference.

\section{Results}

Expression and silencing of HPV-11 L1 in vaginal epithelial cells.HPV-11 L1 mRNA expression in vaginal epithelial cells was analyzed by using RT-qPCR analysis (Fig. 1). Following infection of vaginal epithelial cells, mRNA expression of HPV-11 L1 was significantly increased compared with that in the control group $(\mathrm{P}<0.05)$. In addition, mRNA expression of HPV-11 L1 was significantly decreased in vaginal epithelial cells silenced by siRNA-L1 compared with that in scrambled siRNA-transfected cells as negative controls $(\mathrm{P}<0.05)$.

ELISA was performed to assess the expression of L1 protein in vaginal epithelial cells transfected with siRNA-L1 or scramble siRNA (Fig. 2). L1 protein expression was significantly increased following transfection of vaginal epithelial cells with HPV-11 L1 compared with that in the control group $(\mathrm{P}<0.05)$. In addition, $\mathrm{L} 1$ expression was significantly decreased in vaginal epithelial cells silenced by siRNA-L1 compared with that in the positive and negative controls.

siRNA-HPV-11 L1 decreases the proliferation of vaginal epithelial cells. The MTT assay was performed to analyze the effect of L1 expression on vaginal epithelial cell proliferation (Fig. 3). Following transfection of the cells with HPV-11 L1, the proliferation was markedly decreased compared with that in the control group. However, at day 7 , the proliferation of

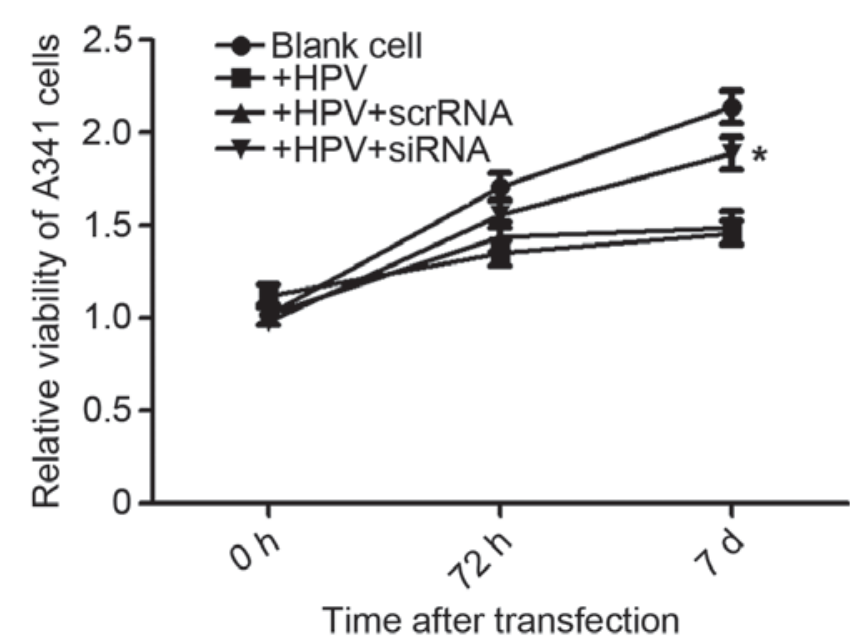

Figure 3. Effects of HPV-11 L1 protein expression on vaginal epithelial cell proliferation. HPV-11 L1 expression inhibited the proliferation of vaginal epithelial cells with time, while this effect was reversed by silencing of L1. ${ }^{*} \mathrm{P}<0.05$ vs. the HPV+scrRNA treated cells. HPV, human papillomavirus; siRNA, small interfering RNA; scrRNA, scrambled siRNA.

vaginal epithelial cells was significantly increased following transfection with siRNA-HPV-11 L1 compared with that of cells transfected with siRNA-scramble $(\mathrm{P}<0.05)$.

Expression of caspase-3 and caspase-9 in vaginal epithelial cells. Caspase-3 and caspase-9 expression levels were assessed to analyze epithelial cell apoptosis (Fig. 4). Caspase-3 and caspase- 9 expression were significantly increased in vaginal epithelial cells transfected with HPV-11 L1 compared with that in the control group $(\mathrm{P}<0.05)$. Following transfection with siRNA-L1, caspase-3 and caspase-9 expression levels were significantly decreased compared with those in the positive and negative controls $(\mathrm{P}<0.05)$. 

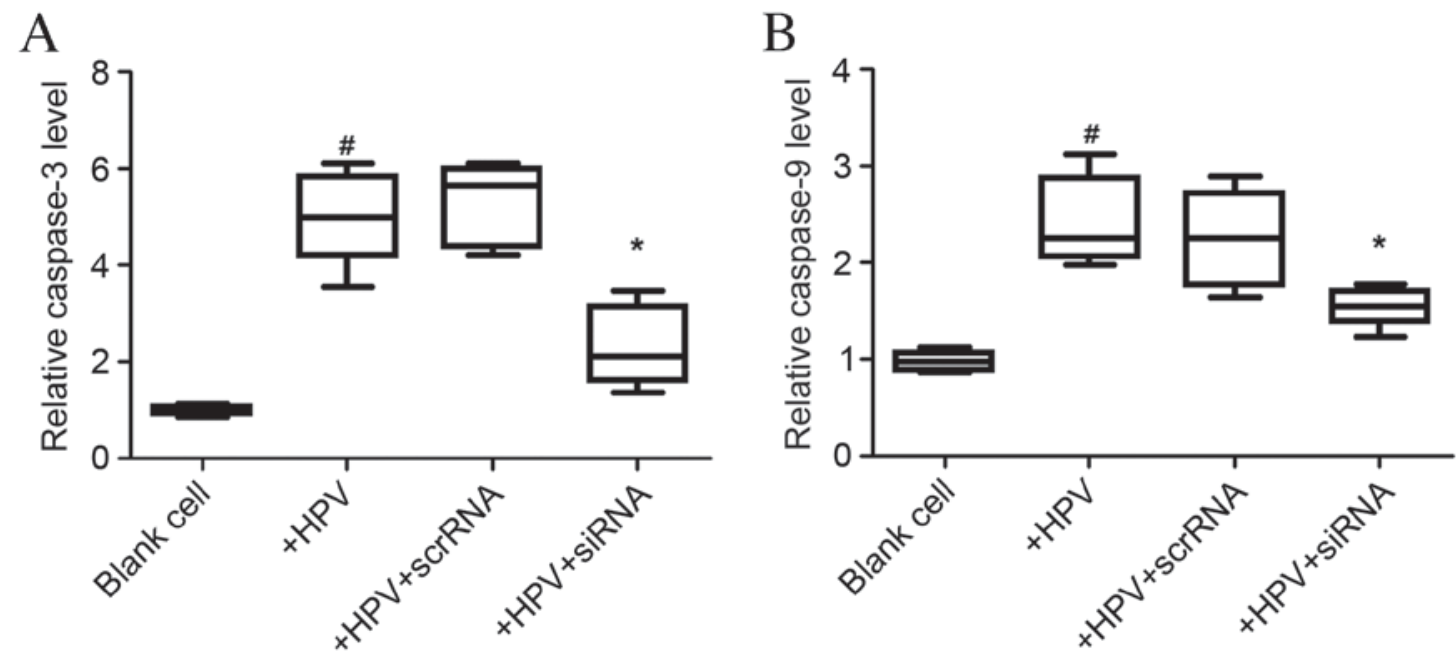

Figure 4. Influence of HPV-11 L1 expression on apoptosis-associated protein expression in vaginal epithelial cells. HPV-11 L1 overexpression significantly increased (A) caspase-3 and (B) caspase-9 expression compared with the blank control group. This effect was significantly reversed by silencing of L1. The boxplots encompass data points between the first and third quartiles. The mean is indicated by a horizontal line, Whiskers extend to $1.5 \mathrm{x}$ interquartile range. Error bar indicates maximum or minimum extension. ${ }^{\#} \mathrm{P}<0.05$, vs. the blank cell, ${ }^{*} \mathrm{P}<0.05$ vs. the HPV+scrRNA treated cells. HPV, human papillomavirus; siRNA, small interfering RNA; scrRNA, scrambled siRNA.

\section{Discussion}

HPV is the most common sexually transmitted virus in humans and an increasing number of studies have reported its significant impact on various typical gynecological malignancies, including cervical cancer and vaginitis $(20,21)$. At present, few effective treatments or preventative methods have been identified due to the complex pathogenesis and type diversity of HPV. The present study evaluated the effects of HPV-11 L1 on the cell bioactivity of vaginal epithelial cells via gene silencing method. The results demonstrated that HPV-11 L1 transfection significantly decreased vaginal epithelial cell proliferation and induced the expression of the apoptotic proteins caspase- 3 and caspase-9.

At present, the target genes typically selected for genetic engineering for HPV prevention are L1 and L2 (22), whereas E6 and E7 are the major targets for HPV therapeutic genetic engineering (23). It has been demonstrated that HPV-11 L1, which is encoded by the HPV-11 L1 gene, was the major capsid protein that was able to self-assemble in vitro and in vivo (24). HPV-11 infection may destroy normal vaginal tissues by suppressing cell proliferation and inducing apoptosis (25). The present study demonstrated that siRNA HPV-11 L1 significantly increased vaginal epithelial cell proliferation, indicating that silencing of HPV-11 L1 may have protective roles in the HPV 11 L1-infected cell proliferation process.

Caspase-3 and caspase-9 levels were significantly decreased in L1-silenced cells. Increased caspase-3 and caspase-9 levels are known as indicators of cell apoptosis $(26,27)$ and a recent study revealed that HPV-16 infection induced cell apoptosis of cervical cancer cells (28). Furthermore, HPV-11 infection was demonstrated to be significantly correlated with several gynecological diseases (29). The present study demonstrated that the high level of HPV-11 L1 significantly increased caspase-3 and caspase-9 expression, indicating that HPV-11 L1 infection may contribute to disease progression. However, when HPV-11 L1 expression was silenced, the levels of caspase proteins were significantly downregulated; therefore it was hypothesized that silencing of HPV-11 L1 may be beneficial for patients with HPV infection diagnosed with vaginitis.

In conclusion, the results of the present study suggested that silencing of HPV-11 L1 expression may reduce the harmful effects of HPV-11 infection on vaginal epithelial cells by increasing the proliferative ability and inhibiting apoptosis. HPV-11 infection may contribute to the development of vaginitis by decreasing cell proliferation and inducing cell apoptosis; however, these effects may be reversed by silencing of L1. The findings of the present study provide a basic exploration of therapeutic and preventative methods to reduce the impact of HPV on gynecological diseases in a clinical setting.

\section{References}

1. Machado-de-Sena RM, Corrêa L, Kato IT, Prates R, Senna AM, Santos CC, Picanço DA and Ribeiro MS: Photodynamic therapy has antifungal effect and reduces inflammatory signals in Candida albicans-induced murine vaginitis. Photodiagnosis Photodyn Ther 11: 275-282, 2014.

2. Olowe O, Makanjuola O, Olowe R and Adekanle D: Prevalence of vulvovaginal candidiasis, trichomoniasis and bacterial vaginosis among pregnant women receiving antenatal care in Southwestern Nigeria. Eur J Microbiol Immunol (Bp) 4: 193-197, 2014.

3. Mayer BT, Srinivasan S, Fiedler TL, Marrazzo JM, Fredricks DN and Schiffer JT: Rapid and profound shifts in the vaginal microbiota following antibiotic treatment for bacterial vaginosis. J Infect Dis 212: 793-802, 2015.

4. Lester J, Pahouja G, Andersen B and Lustberg M: Atrophic vaginitis in breast cancer survivors: A difficult survivorship issue. J Personal Med 5: 50-66, 2015.

5. Olowe OA, Makanjuola OB, Olowe R and Adekanle DA: Prevalence of vulvovaginal candidiasis, trichomoniasis and bacterial vaginosis among pregnant women receiving antenatal care in Southwestern Nigeria. Eur J Microbiol Immunol (Bp) 4: 193-197, 2014.

6. Keegan H, Pilkington L, Jordan R, Mozes J, Varga N, Benczik M, Riegger L, Kolta P, Sauer U, Preininger C, et al: Human Papillomavirus DNA and Host Gene mRNA Biomarker Detection in a 'Micro Total Analysis System' (microTAS) Device to Aid Diagnosis of Cervical Intraepithelial Neoplasia. In: Proceedings of the United States and Canadian Association of Pathology Annual Meeting, San Diego, CA, 2014. 
7. Thomsen LT, Frederiksen K, Munk C, Junge J, Castle PE, Iftner T and Kjaer SK: High-risk and low-risk human papillomavirus and the absolute risk of cervical intraepithelial neoplasia or cancer. Obstet Gynaecol 123: 57-64, 2014

8. Kreimer AR, González P, Katki HA, Porras C, Schiffman M Rodriguez AC, Solomon D, Jiménez S, Schiller JT, Lowy DR, et al: Efficacy of a bivalent HPV 16/18 vaccine against anal HPV 16/18 infection among young women: A nested analysis within the Costa Rica Vaccine Trial. Lancet Oncol 12: 862-870, 2011.

9. Shah KV: A case for immunization of human papillomavirus (HPV) 6/11-infected pregnant women with the quadrivalent HPV vaccine to prevent juvenile-onset laryngeal papilloma. J Infect Dis 209: 1307-1309, 2014.

10. Gillet E, Meys JF, Verstraelen H, Bosire C, De Sutter P, Temmerman M and Broeck DV: Bacterial vaginosis is associated with uterine cervical human papillomavirus infection: A meta-analysis. BMC Infect Dis 11: 10, 2011.

11. Lazenby GB, Taylor PT, Badman BS, Mchaki E, Korte JE, Soper DE and Young Pierce J: An association between Trichomonas vaginalis and high-risk human papillomavirus in rural Tanzanian women undergoing cervical cancer screening. Clin Ther 36: 38-45, 2014.

12. Weihua Q: Use of eg 2,4-dihydroxybenzoic acid or 3,4,5-trihydroxybenzoic acid or esters thereof; hepatitis B virus (HBV), papillomavirus and/or herpes virus. Google Patents, 2011.

13. Banura C, Mirembe FM, Orem J, Mbonye AK, Kasasa S and Mbidde EK: Prevalence, incidence and risk factors for anogenital warts in Sub Saharan Africa: A systematic review and meta analysis. Infect Agent Cancer 8: 27, 2013.

14. Hillemanns P, Jentschke M, Evans TG, Soergel P and Hass R: Detection of E6-AP as a potential therapeutic target in cervical specimen of HPV-infected women. Arch Gynecol Obstet 289: 1281-1286, 2014

15. Gao G, Chernock RD, Gay HA, Thorstad WL, Zhang TR Wang H, Ma XJ, Luo Y, Lewis JS Jr and Wang X: A novel RT-PCR method for quantification of human papillomavirus transcripts in archived tissues and its application in oropharyngeal cancer prognosis. Int J Cancer 132: 882-890, 2013.

16. Livak KJ and Schmittgen TD: Analysis of relative gene expression data using real-time quantitative PCR and the 2(-Delta Delta C(T)) method. Methods 25: 402-408, 2001.

17. Apilux A, Ukita Y, Chikae M, Chailapakul O and Takamura Y: Development of automated paper-based devices for sequential multistep sandwich enzyme-linked immunosorbent assays using inkjet printing. Lab Chip 13: 126-135, 2013.
18. Cullen B, Henriksen B and Lomneth R: Structural aspects of ozonides on lymphoma cell viability: Part B. J Chem Pharm Res 6: 329-334, 2014.

19. Hou X, Yu Q, Zeng F, Yu C and Wu S: Ratiometric fluorescence assay for $\gamma$-glutamyltranspeptidase detection based on a single fluorophore via analyte-induced variation of substitution. Chem Commun 50: 3417-3420, 2014.

20. Doorbar J, Egawa N, Griffin H, Kranjec C and Murakami I: Human papillomavirus molecular biology and disease association. Rev Med Virol 25 (Suppl 1): S2-S23, 2015.

21. Rimawi BH, Mirdamadi M and John JF Jr: Infections and homelessness: Risks of increased infectious diseases in displaced women. Word Med Health Policy 6: 118-132, 2014.

22. Jagu S, Kwak K, Garcea RL and Roden RB: Vaccination with multimeric L2 fusion protein and L1 VLP or capsomeres to broaden protection against HPV infection. Vaccine 28: 4478-4486, 2010.

23. Welters MJ, Kenter GG, Piersma SJ, Vloon AP, Löwik MJ, Berends-van der Meer DM, Drijfhout JW, Valentijn AR, Wafelman AR, Oostendorp J, et al: Induction of tumor-specific CD4+ and CD8+ T-cell immunity in cervical cancer patients by a human papillomavirus type 16 E6 and E7 long peptides vaccine. Clin Cancer Res 14: 178-187, 2008.

24. Li M, Cripe TP, Estes PA, Lyon MK, Rose RC and Garcea RL: Expression of the human papillomavirus type $11 \mathrm{~L} 1$ capsid protein in Escherichia coli: Characterization of protein domains involved in DNA binding and capsid assembly. J Virol 71: 2988-2995, 1997.

25. Fage SW, Iftner T, Thyssen JP and Kjaer SK: Filaggrin, human papillomavirus and the cervix. In: Filaggrin. Berlin Heidelberg, Springer, pp333-342, 2014.

26. Porter AG and Jänicke RU: Emerging roles of caspase-3 in apoptosis. Cell Death Differ 6: 99-104, 1999.

27. Simbulan-Rosenthal CM, Velena A, Veldman T, Schlegel R and Rosenthal DS: HPV-16 E6/7 immortalization sensitizes human keratinocytes to ultraviolet $\mathrm{B}$ by altering the pathway from caspase-8 to caspase-9-dependent apoptosis. J Biol Chem 277: 24709-24716, 2002

28. Tommasino M: The human papillomavirus family and its role in carcinogenesis. Semin Cancer Biol 26: 13-21, 2014.

29. Hougardy BM, van der Zee AG, van den Heuvel FA, Timmer T, de Vries EG and de Jong S: Sensitivity to Fas-mediated apoptosis in high-risk HPV-positive human cervical cancer cells: Relationship with Fas, caspase-8, and Bid. Gynecol Oncol 97: 353-364, 2005 . 